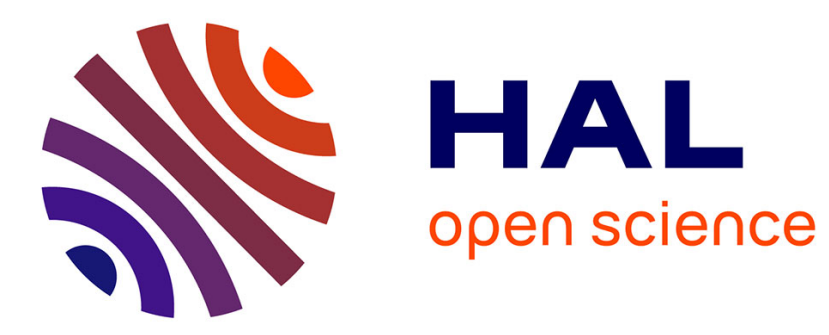

\title{
High Gain Observer for Backstepping Control of a MRI-guided Therapeutic Microrobot in Blood Vessels
}

Laurent Arcèse, Ali Cherry, Matthieu Fruchard, Antoine Ferreira

\section{To cite this version:}

Laurent Arcèse, Ali Cherry, Matthieu Fruchard, Antoine Ferreira. High Gain Observer for Backstepping Control of a MRI-guided Therapeutic Microrobot in Blood Vessels. 2010 3rd IEEE RAS and EMBS International Conference on Biomedical Robotics and Biomechatronics (BioRob), Sep 2010, Tokyo, Japan. pp 349 - 354, 10.1109/BIOROB.2010.5627806 . hal-00837711

\section{HAL Id: hal-00837711 https://hal.science/hal-00837711}

Submitted on 24 Jun 2013

HAL is a multi-disciplinary open access archive for the deposit and dissemination of scientific research documents, whether they are published or not. The documents may come from teaching and research institutions in France or abroad, or from public or private research centers.
L'archive ouverte pluridisciplinaire HAL, est destinée au dépôt et à la diffusion de documents scientifiques de niveau recherche, publiés ou non, émanant des établissements d'enseignement et de recherche français ou étrangers, des laboratoires publics ou privés. 


\title{
High Gain Observer for Backstepping Control of a MRI-guided Therapeutic Microrobot in Blood Vessels
}

\author{
Laurent Arcese, Ali Cherry, Matthieu Fruchard, Antoine Ferreira
}

\begin{abstract}
This paper reports precise modeling and controller/observer design for a microsized polymer aggregate of magnetic particles inside an artery, using a Magnetic Resonance Imaging (MRI) device for supplying propulsion in order to achieve targeted chemotherapy. Non-Newtonian behaviour of blood is taken into account, as well as wall effects and interactions, resulting in a highly nonlinear model. A High Gain Observer is designed to reconstruct the unmeasured state, so that a backstepping controller can be synthesized. Efficiency and robustness to noise of this controller/observer pair is then illustrated through simulations results.
\end{abstract}

\section{INTRODUCTION}

Minimally invasive cancer therapy is currently an active research area since related techniques can both reach remote places without operating and reduce drug or radiation dose. Thereby, they result in lessened medical side effects and a better therapeutic efficiency and safety. Among these approaches, cathetered embolization, focused ultrasound or radiotherapy [1] are commonly used, though restricted to accessible places or limited by the non ability to target precisely tumor cells. These drawbacks can be avoided by targeted chemotherapy, which relies on a selective delivery using either drugs linked to antibodies specific for tumorassociated antigenes [2], or drugs carried by autonomous untethered microrobots controlled so as to reach the tumor. However, it proves difficult to embed actuators sufficiently powerful to propel such robots in the cardiovascular system, especially when swimming against the blood flow. Most of swimming approaches consequently rely upon magnetic fields to wirelessly transmit power to the microrobot [3], [4], [5].

A recent magnetic actuation, particularly well-suited for medical purposes, has been provided by Martel [6]. The authors improve the classical bead pulling through the use of the gradients coils of clinical MRI devices to provide propelling power. On top of that, the relevance of using clinical MRI devices is twofold: they are widely spread in hospitals, and their imaging ability yields fine

This work was supported by European Union's 7th Framework Program and its research area ICT-2007.3.6 Micro/nanosystems under the project NANOMA (Nano-Actuactors and Nano-Sensors for Medical Applications).

L. Arcese and M. Fruchard are with the Institut PRISME UPRES 4229 , IUT de Bourges, 63 Av de Lattre de Tassigny, 18020, Bourges cedex, France laurent.arcesedbourges.univ-orleans.fr matthieu.fruchardabourges.univ-orleans.fr

A. Cherry and A. Ferreira are with the Institut PRISME UPRES 4229, ENSI de Bourges, 88 Bd Lahitolle, 18000, Bourges, France ali.cherrydensi-bourges.fr antoine.ferreira@ensi-bourges.fr observation of the robot's position in the cardiovascular system. The control and imaging multiplexing has already been experimented in [7].

As the smallest capillaries are under a half-dozen micrometers large, the size of the robot should not exceed 100 nanometers [8] to avoid embolization hazards and to drive the drugs as close as possible to the tumor. However, forces induced by clinical MRI coils are not strong enough to steer nanorobots in larger vessels. Besides, hydrodynamics wall effects show that a partial vessel occlusion by the robot results in an optimal ratio between the robot's and the vessel's radii [6], [9]. A natural way of overcoming the MRI limitations is thus to make the radius of the robot decrease as it enters smaller vessels, e.g. with a microrobot made of a degradable polymer binded aggregate of magnetic particles.

We first define a precise forces balance (Section II), which includes wall effects (parabolic profile of blood flow, pulsatile arterial walls and effect of the ratio of robot's on vessel's radii), wall interactions (Van der Waals and electrostatic forces) and non-Newtonian behaviour of blood. Although this forces balance results in a highly nonlinear model (even if considering the sole hydrodynamics forces), the great majority of related literature, to our knowledge, synthesizes control laws using linear tools. Besides, there is no consideration about observation issues. Hence, instabilities or sensitivity to noise or to unmodeled dynamics, reported for instance in [7] with a PID controller, could have been expected. We show that a backstepping control approach (Section III), designed for this specific non linear model, improves the quality and robustness of the tracking along a reference trajectory. As the MRI imager only measures the robot's position, we develop a high gain observer (Section IV) to rebuild the microrobot's velocity, which is required to implement the control law. In Section V, simulation experiments illustrate the benefits of using this controller-observer pair in an artery with minor bifurcations. In particular, the high gain observer, designed so as to ensure robustness to noise measurement, greatly smooth the output and in turn improves the overall tracking efficiency. Finally, conclusion and prospects are discussed in the last section.

\section{Modeling}

The robot is a polymer binded aggregate of ferromagnetic particles immersed in blood vessels. A 2D nonlinear model of the robot is established, which encompasses the different forces that affect the robot's motion as well as its 
interactions with the vessel's walls. We will then infer from this model the existence of an optimal trajectory, and the synthesis of a control law along this trajectory.

The translational motion of the robot is expressed by:

$$
m \frac{d \vec{v}}{d t}=\vec{F}_{m}+\vec{F}_{d}+\vec{W}_{a}+\vec{F}_{v d w}+\vec{F}_{\text {elec }}
$$

where $\vec{v}$ is the translational velocity of the robot and $m$ its mass. $\vec{F}_{m}, \vec{F}_{d}$ and $\vec{W}_{a}$ respectively denote the magnetic force produced by the MRI gradient coils, blood hydrodynamic drag force and apparent weight. $\vec{F}_{v d w}$ and $\vec{F}_{\text {elec }}$ are the Van der Waals and the electrostatic interaction forces between the robot and the vessel's walls.

In the rest of this paper, we assume that the orientation of the robot does not change due to the magnetic torque which tends to align the magnetization of the robot along the external MRI field $\left(B_{0}=1.5 T\right)$. We also assume that the robot is large enough to neglect the effect of Brownian motion.

\section{A. Hydrodynamic drag force}

The hydrodynamic drag force $\vec{F}_{d}$ exerting on a spherical body is expressed as:

$$
\vec{F}_{d}=-\frac{1}{2} \rho_{f}\left[\frac{\left\|\left(\vec{v}-\vec{v}_{f}\right)\right\|}{\beta}\right]^{2} A C_{d} \frac{\left(\vec{v}-\vec{v}_{f}\right)}{\left\|\left(\vec{v}-\vec{v}_{f}\right)\right\|}
$$

where $\vec{v}-\vec{v}_{f}$ denotes the relative velocity of the robot with respect to the fluid, $A$ is the frontal area of the core, $\rho_{f}$ is the density of the fluid, and $\beta$ a dimensionless ratio related to wall effect caused by the vessel's occlusion by the robot [10]:

$$
\beta=\frac{1+\left(\frac{\lambda}{\lambda_{0}}\right)^{\alpha_{0}}}{1-\lambda^{\alpha_{0}}}
$$

with ratio $\lambda=2 r / D$ and $D$ denoting the vessel's diameter (in meter). Parameters $\alpha_{0}$ and $\lambda_{0}$ are functions of the Reynolds number, but are commonly set to 1.5 and 0.29 , respectively. The drag coefficient $C_{d}$, which is a function of the Reynolds number $R e=\frac{2 r \rho_{f}\left|\vec{v}-\vec{v}_{f}\right|}{\beta \eta}$, is given by [11]:

$$
C_{d}=\frac{24}{R e}+\frac{6}{1+\sqrt{R e}}+0.4
$$

In the case of blood, which exhibits a non-newtonian behavior, the fluid's viscosity $\eta$ is a function of vessel's diameter $d$ (in micron) and hematocrit rate $h_{d}$ according to the three following empirical relationships [12]:

$$
\eta=\frac{\eta_{\text {plasma }} d^{2}}{(d-1.1)^{2}}\left[1+\frac{\left(\eta_{0.45}-1\right) d^{2}}{(d-1.1)^{2}} \frac{\left(1-h_{d}\right)^{c}-1}{(1-0.45)^{c}-1}\right]
$$

with parameters $\eta_{\text {plasma }}$ and $\eta_{0.45}$ denoting respectively the plasma's viscosity and the relative apparent blood's viscosity for a fixed discharge hematocrit of 0.45 , given by:

$$
\eta_{0.45}=6 e^{-0.085 d}+3.2-2.44 e^{-0.06 d^{0.645}}
$$

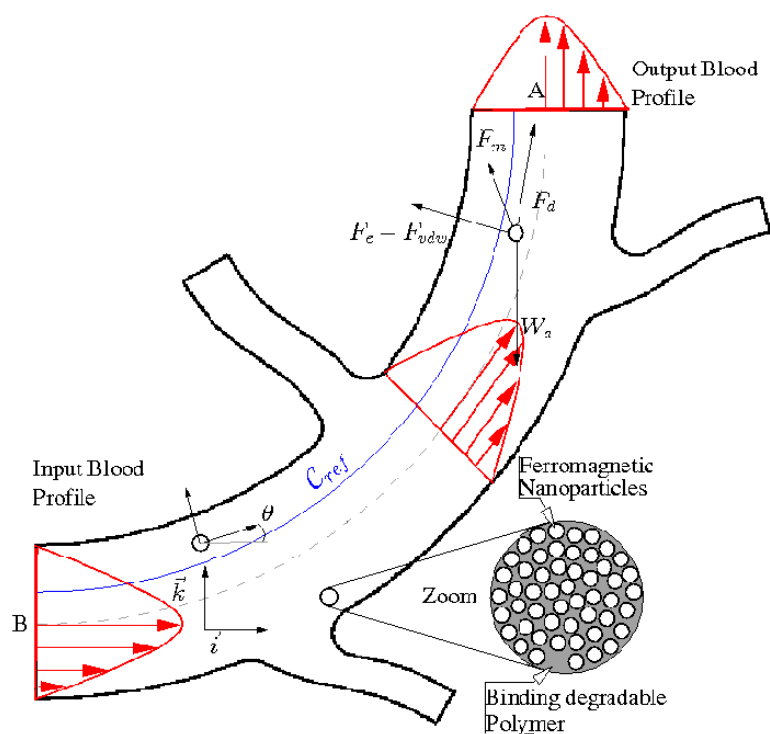

Fig. 1. Scheme of a blood vessel with minor bifurcations

The shape of the viscosity dependance on hematocrit is:

$$
c=\frac{10^{11}}{d^{12}}-\left(0.8+e^{-0.075 d}\right)\left(\frac{d^{12}}{d^{12}+10^{11}}\right)
$$

Wall effects of the vessel on the fluid traditionally result in a parabolic profile of blood flow (see Figure 1). Besides, to fully take into account pulsatile flow caused by heart pumping in arteries, we also consider a periodic deformation of the vessel's diameter $D(t)$ synchronized with the pulsative blood's velocity $v_{f}(t)$.

\section{B. Apparent weight}

In addition to the hydrodynamic force, apparent weight (combined action of weight and buoyancy) is acting on the robot:

$$
\vec{W}_{a}=V\left(\rho-\rho_{f}\right) \vec{g}
$$

where $\rho=\tau_{m} \rho_{m}+\left(1-\tau_{m}\right) \rho_{\text {poly }}$ with $\rho_{m}$ and $\rho_{\text {poly }}$ the magnetic material's and polymer's densities.

\section{Van der Waals and electrostatic forces}

The robot and the wall interact each other through Van der Waals and electrostatic forces. The Van der Waals potential between the spherical robot and the wall is given by [13]:

$$
\vec{V}_{v d w}=-\frac{A_{h}}{6}\left(\frac{1}{H}+\frac{1}{2+H}+\ln \frac{h}{2+H}\right) \vec{n}
$$

where $A_{h}$ is the Hamaker constant and $H=h / r$ is the normalized distance between the robot and the wall. Then, the Van der Waals interaction force is given by differenciating (4): $\vec{F}_{v d w}=-\nabla \vec{V}_{v d w}$.

The electrostatic force between the robot and the wall considered as an uncharged surface is given by [14]:

$$
\vec{F}_{\text {elec }}=\frac{q^{2}}{4 \pi \epsilon \epsilon_{0}(r+h)^{2}} \vec{n}
$$


with $q$ the robot's charge, $\epsilon$ the dielectric density of the medium in which the interaction occurs and $\epsilon_{0}$ the vacuum permittivity. [15] gives the expression of the maximum allowable charge for a spherical body of radius $r$ :

$$
q(\mu C)=S \times Q=4 \pi r^{2} \times 30(100 r)^{-0.3}
$$

\section{Magnetic force}

The gradient coils of the MRI system provide magnetic gradients which produce a magnetic force $F_{m}$ on the robot:

$$
\vec{F}_{m}=\tau_{m} \mu_{0} V(\vec{M} \cdot \nabla) \vec{H}
$$

where $V$ is the robot's total volume, $\tau_{m}=\frac{V_{m}}{V}$ with $V_{m}$ the ferromagnetic volume, $\vec{M}$ is the magnetization of the material, $\mu_{0}$ is the permeability of free space and $\vec{H}$ is the external magnetic field. Note that $B_{0}$ is sufficient to ensure a saturation magnetization $M_{\text {sat }}$.

\section{E. State Space Representation}

Let $(x, z)$ denote the position of the robot in the vessel with respect to a given frame $\mathcal{F}(O, \vec{i}, \vec{k})$. The state model is established from differential equation (1) defining the robot's dynamic behavior, projected on $\vec{i}$ and $\vec{k}$ axes:

$$
\left\{\begin{array}{l}
m \ddot{x}=F_{m_{x}}+F_{d_{x}}+F_{v d w_{x}}+F_{\text {elec }_{x}} \\
m \ddot{z}=F_{m_{z}}+F_{d_{z}}+W_{a}+F_{v d w_{z}}+F_{\text {elec }_{z}}
\end{array}\right.
$$

where indexes $x$ (respectively $z$ ) denote projections on $\vec{i}(\vec{k})$.

Let $x_{1}, x_{2},\left(x_{3}, x_{4}\right)$ denote respectively the particule's position and velocity along $\vec{i}$ axis (respectively along $\vec{k}$ axis). Assuming that positions $x_{1}$ and $x_{3}$ can be measured thanks to the MRI imaging system, let $y$ denotes the state's measure. Using expressions of forces given by (6), (2), (3), (5) and Van der Waals interaction derivated from potential (4), and adequate projection of local frame along the geometry of the vessel, system (7) can be written in the form:

$$
(\mathcal{S})\left\{\begin{array}{l}
\dot{x}_{1}=x_{2} \\
\dot{x}_{2}=f_{2}\left(x_{1}, x_{2}, x_{3}\right)+a u_{1} \\
\dot{x}_{3}=x_{4} \\
\dot{x}_{4}=f_{4}\left(x_{1}, x_{3}, x_{4}\right)+a u_{2} \\
y=\left(x_{1}, x_{3}\right)^{T}
\end{array}\right.
$$

where control inputs $u_{1}=\nabla B_{x}$ and $u_{2}=\nabla B_{z}$ are the magnetic gradients, parameter $a=\frac{\tau_{m} M}{\rho}$, and $f_{i}$ are highly nonlinear functions.

\section{CONTROL APPROACH}

From the previous force balance, an optimal trajectory is defined so as to minimize the control efforts [16]. This trajectory passes through the point where the electrostatic and Van der Waals forces counterbalance perfectly the robot's weight. This reference trajectory is closed to the vessel's wall since the drag force decreases due to the parabolic profile.

A control law Lyapunov-stabilizing trajectories for system $(\mathcal{S})$ is now presented. The determination of Lyapunov functions is generically a challenging issue. According to
[17], [18], it is preferable to use control Lyapunov functions (CLF) in a backstepping control approach. Since this design requires a triangular form for the control system, we propose the following change of variables:

$$
\left\{X=\left(\begin{array}{c}
x_{1} \\
x_{3}
\end{array}\right) ; \quad Z=\left(\begin{array}{c}
x_{2} \\
x_{4}
\end{array}\right) ; \quad U=a\left(\begin{array}{l}
u_{1} \\
u_{2}
\end{array}\right)\right.
$$

Thus, we obtain a new system in a triangular form:

$$
\left(\mathcal{S}^{\prime}\right)\left\{\begin{array}{l}
\dot{X}=Z \\
\dot{Z}=F(X, Z)+U \\
Y=X
\end{array}\right.
$$

Using the change of variables given in (9), we build in two steps a control law from assignable Lyapunov function.

Step 1: Let

$$
\left\{\begin{array}{l}
\tilde{X}=X_{\text {ref }}-X \\
\tilde{Z}=Z_{\text {ref }}-Z
\end{array}\right.
$$

respectively denote the position and velocity error. $X_{r e f}$, $\dot{X}_{\text {ref }}=Z_{\text {ref }}$ and $\ddot{X}_{r e f}$ are respectively the desired reference trajectory, velocity and acceleration.

The CLF candidate is given by:

$$
V_{1}(\tilde{X})=\frac{1}{2} \tilde{X}^{T} \tilde{X} \quad \geq 0
$$

Calculating the derivative of $V_{1}(\tilde{X})$ along system (10), we have:

$$
\dot{V}_{1}(\tilde{X})=\frac{\partial V_{1}(\tilde{X})}{\partial \tilde{X}} \dot{\tilde{X}}=\tilde{X}^{T}\left(\dot{X}_{\text {ref }}-Z\right)
$$

To ensure $\dot{V}_{1}(\tilde{X})$ definite negative, we set:

$$
\dot{V}_{1}(\tilde{X})=-k_{1} \tilde{X}^{T} \tilde{X}, \quad k_{1} \geq 0
$$

From (11), (13) and (14), we obtain:

$$
\dot{\tilde{Z}}=\ddot{X}_{\text {ref }}+k_{1} \tilde{Z}-k_{1}^{2} \tilde{X}-\dot{Z}
$$

Step 2: In this step, the CLF is given by:

$$
V_{2}(\tilde{X}, \tilde{Z})=V_{1}(\tilde{X})+\frac{1}{2} \tilde{Z}^{T} \tilde{Z} \quad \geq 0
$$

The derivative of $V_{2}(\tilde{X}, \tilde{Z})$ along system (10) is expressed as:

$$
\dot{V}_{2}(\tilde{X}, \tilde{Z})=\tilde{X}^{T} \dot{\tilde{X}}+\tilde{Z}^{T} \dot{\tilde{Z}}
$$

From (11), (15) and (17), we obtain:

$$
\begin{aligned}
\dot{V}_{2}(\tilde{X}, \tilde{Z})= & -k_{1} \tilde{X}^{T} \tilde{X}+\tilde{Z}^{T}\left(\left(1-k_{1}^{2}\right) \tilde{X}+k_{1} \tilde{Z}\right) \\
& +\tilde{Z}^{T}\left(\ddot{X}_{r e f}-\dot{Z}\right)
\end{aligned}
$$

Likewise, to ensure $\dot{V}_{2}(\tilde{X}, \tilde{Z})$ definite negative, we set:

$$
\dot{V}_{2}(\tilde{X}, \tilde{Z})=-k_{1} \tilde{X}^{T} \tilde{X}-k_{2} \tilde{Z}^{T} \tilde{Z}, \quad k_{2} \geq 0
$$

From (10), (11), (18) and (19), the control law can be expressed as:

$$
\begin{aligned}
U= & \ddot{X}_{r e f}+\left(k_{1}+k_{2}\right) \dot{X}_{r e f}+\left(1+k_{1} k_{2}\right) X_{r e f} \\
& -\left(1+k_{1} k_{2}\right) X-\left(k_{1}+k_{2}\right) Z-F(X, Z)
\end{aligned}
$$




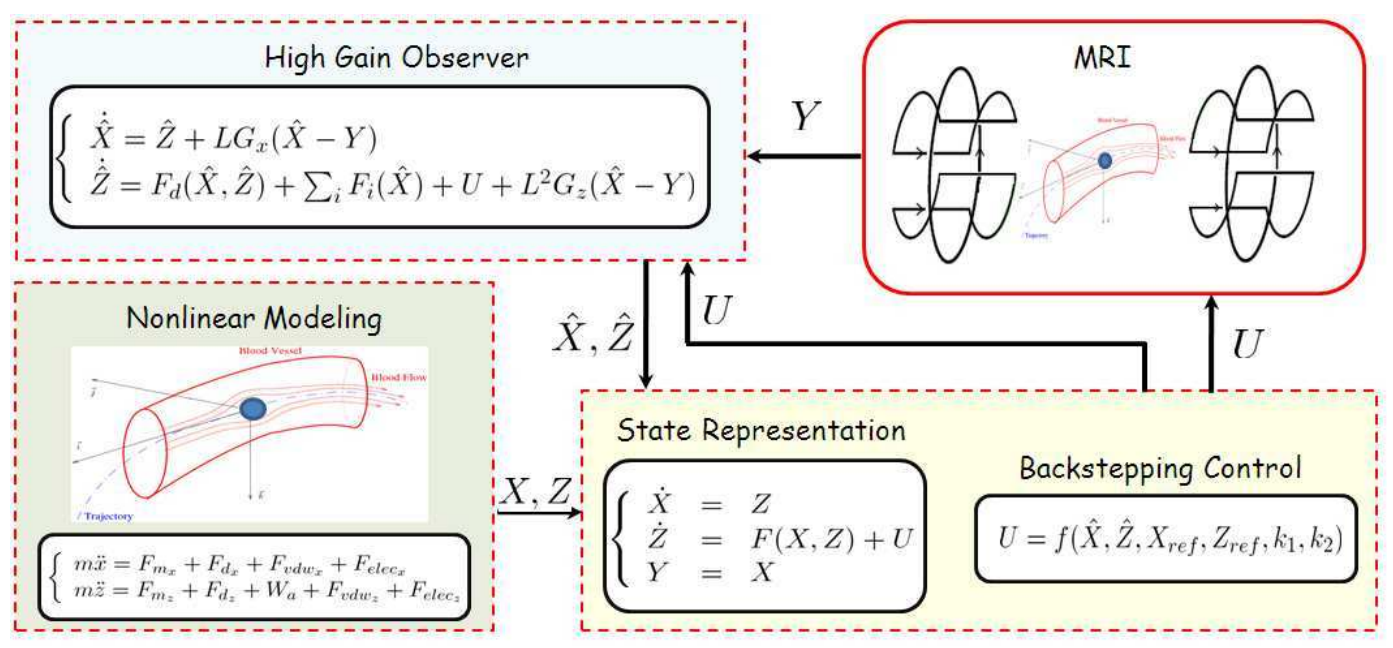

Fig. 2. Closed-loop system overview

\section{HIGH GAIN OBSERVER}

The backstepping control law (20) requires the knowledge of the robot's velocity in addition to its position, measured by the MRI system. Among the different observers cited in the literature, our attention was drawn to the high gain observer. Unlike other observers, locally Lipschitzian state's functions ensure the convergence of the observer. This one requires the system to have canonical form which can be obtained using the change of variables of equation (9) leading to system (10), where the function $F(X, Z)$ can be split up into two parts as follows:

$$
F(X, Z)=F_{d}(X, Z)+\sum_{i} F_{i}(X)
$$

From the forces expressions of section II, one can notice that only the drag force depends on the robot's position and velocity. Hence, only drag force has to be locally Lipschitzian to ensure the convergence of the observer given by [19]:

$$
\left\{\begin{array}{l}
\dot{\hat{X}}=\hat{Z}+L G_{x}(\hat{X}-Y) \\
\dot{\hat{Z}}=F_{d}(\hat{X}, \hat{Z})+\sum_{i} F_{i}(\hat{X})+U+L^{2} G_{z}(\hat{X}-Y)
\end{array}\right.
$$

where $L$ is the high gain, $G_{x}=\left(\begin{array}{cc}g_{1} & 0 \\ 0 & g_{3}\end{array}\right)$ and $G_{z}=\left(\begin{array}{cc}g_{2} & 0 \\ 0 & g_{4}\end{array}\right)$ are defined from the Hurwitz matrix H:

$$
H=\left(\begin{array}{cccc}
g_{1} & 0 & 1 & 0 \\
0 & g_{3} & 0 & 1 \\
g_{2} & 0 & 0 & 0 \\
0 & g_{4} & 0 & 0
\end{array}\right)
$$

\section{SIMULATIONS}

The robot is made of $\tau_{m}=80 \% \mathrm{NdFeB}$ particles (high remanence and coercivity) and $20 \%$ of binding polymer. Simulations are led within the limitations of a clinical MRI system. In order not to exceed the capacity of existing MRI systems, the applied control law (20) is now corrected as $\frac{u_{i}}{k(t)}$, with $k(t)=\max \left\{1, \frac{u_{i}}{u_{i, m a x}}\right\}$.

The pulsative blood's velocity is modeled by an affine combination of a time-varying periodic flow with a spatial parabolic shape. So as to simplify the analytical expression, but with no loss of generality, we consider the first terms in the time-varying Fourier series of the physiological pulse. In the case of an artery, such an approximation leads to:

$v_{f}(t)=0.025\left(1+1.15 \sin \left(2 \pi t+\frac{\pi}{2}\right)\right) \times\left[1-\left(\frac{D / 2-h}{r}\right)^{2}\right]$

Our studies assume the presence of minor bifurcations (Figure 1). The controller must be sufficiently robust to compensate the minor changes of blood's flow, considered as a disturbance, they induce. Note that major bifurcations will require a further study of velocity's field profile.

Performances and stability of the controller with respect to noise measurement, parameters variations and uncertainties are now illustrated by simulations, whose nominal parameters are given in Table I.

In the first simulation, we assume that a white gaussian noise set to $10 \%$ of the measured signal is applied on the position measurement. MRI devices currently have a precision around some hundreds of micrometers. Besides, since the microrobot is composed of a ferromagnetic nanoparticles aggregate, it induces artifacts on the MRI imaging. That is why robustness to output noise is a key point for implementation on such process. On the one hand, Figure 3(a) illustrates that the closed-loop system is stable and the tracking is efficient and robust to noise. Furthermore, despite the presence of a $100 \mu \mathrm{m}$ noise amplitude, the estimation error is fewer than $10 \mu m$ (i.e. $10 \%$ of nominal value) after the observer has converged (Figure 3(b)). On the other hand, control inputs are very sensitive to noise (see Figure 4), especially around $t=0.5 \mathrm{~s}$ 

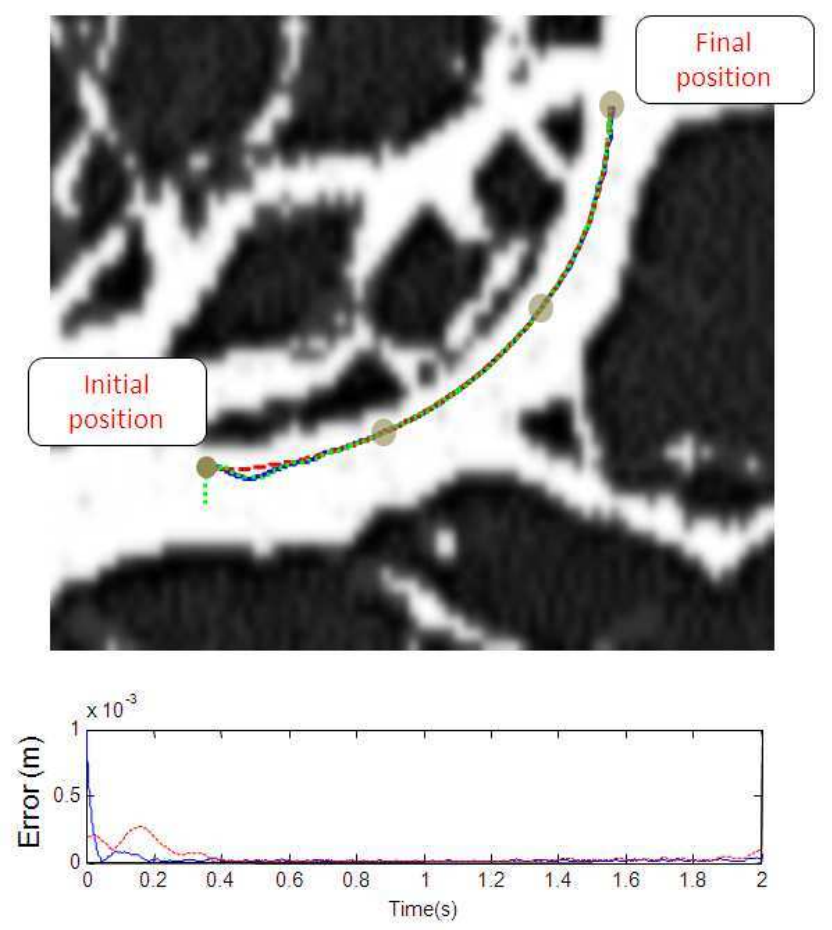

Fig. 3. (a) XZ trajectory: reference trajectory (red dashed line), real trajectory (blue solid line) and estimated trajectory (green dotted line). (b) Error: tracking error (red dashed line) estimation error (blue solid line)

and $t=1.5$ s, i.e. when the blood is flowing back. At these moments, the drag force is not dominant in the force balance, unlike wall's interactions. Yet, these latter are very sensitive to noise on the measured position, whereas drag force is mainly sensitive to error on velocities. Since velocity errors are filtered by the observer, drag estimation is more robust to noise measurements than the precompensation of wall's interactions in the backstepping control law. That is why control inputs are smoother around $t=1 \mathrm{~s}$, when the drag force prevails. The situation is a bit different at $t=2 s$ : despite a maximal blood's flow, the drag force is dominated because the optimal trajectory requires the robot to be close to the wall.

The second simulation is performed while assuming that the blood's viscosity is affected by uncertainties of $10 \%$ of its nominal value. This uncertainty is motivated by the difficulty to estimate accurately biological parameters. Parametric error does not affect the observer's convergence (see Figure 5(b)), and the closed-loop system remains stable (see Figure 5(a)). Nevertheless, we note an important tracking error (Figure 5(b)) closely related to the pumping flow: since the viscosity is overestimated by the controller, the robot does not slow down enough to ensure a fine tracking. Notwithstanding this tracking error, Figure 5(a) shows that the path tracking remains good, which can be expected since uncertainty on the viscosity affects the pre-compensation of the sole drag force in the backstepping approach: the tracking error occurs mainly along the

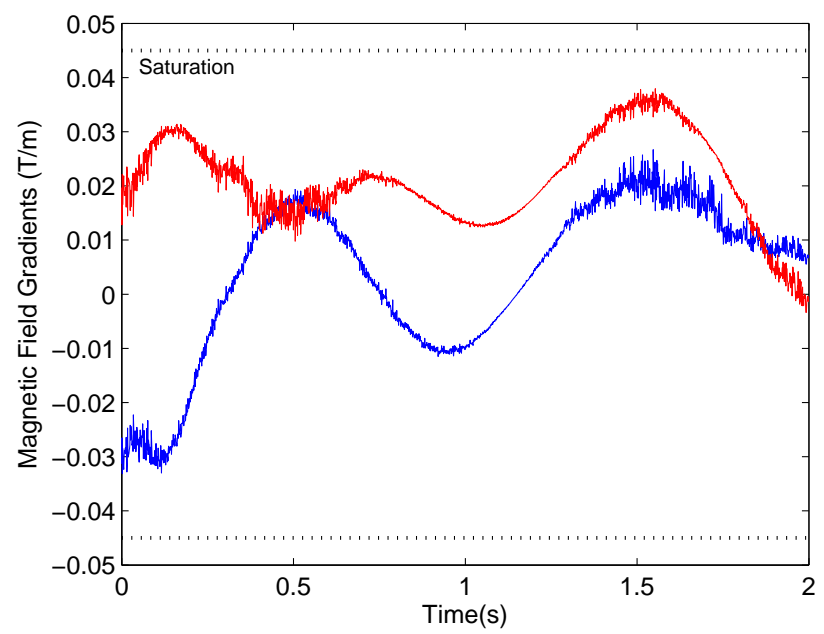

Fig. 4. Control input : magnetic field gradients on $\vec{i}$-axis (red) and on $\vec{k}$-axis (blue)

TABLE I

SIMULATIONS DATA

\begin{tabular}{|c||c|c|}
\hline Plasma's viscosity & $\eta_{\text {plasma }}$ & $5 \times 10^{-3}[\mathrm{~Pa} . \mathrm{s}]$ \\
\hline Blood's density & $\rho_{f}$ & $1060\left[\mathrm{~kg} \cdot \mathrm{m}^{-3}\right]$ \\
\hline Robot's density & $\rho_{m}$ & $8000\left[\mathrm{~kg} \cdot \mathrm{m}^{-3}\right]$ \\
\hline Robot's radius & $r$ & $300[\mathrm{\mu m}]$ \\
\hline Vessel's diameter & $D$ & $3[\mathrm{~mm}]$ \\
\hline Polymer's density & $\rho_{\text {poly }}$ & $1500\left[\mathrm{~kg} . \mathrm{m}^{-3}\right]$ \\
\hline Ferromagnetic ratio & $\tau_{m}$ & 0.8 \\
\hline Magnetization & $M$ & $1.95 \times 10^{6}\left[\mathrm{A.m} \mathrm{m}^{-1}\right]$ \\
\hline Hematocrit & $h_{d}$ & 0.45 \\
\hline Hamaker constant & $A_{h}$ & $4 \times 10^{-19}[\mathrm{~J}]$ \\
\hline Blood's dielectric density & $\varepsilon$ & $77\left[\mathrm{C}^{2} \cdot \mathrm{N}^{-1} \cdot \mathrm{m}^{-2}\right]$ \\
\hline Initial condition on $X, Z$ & $X_{0}, Z_{0}$ & $(0,0001)^{T},(0,0)^{T}$ \\
\hline Initial condition on $\hat{X}, \hat{Z}$ & $\hat{X}_{0}, \hat{Z}_{0}$ & $(0,0)^{T},(0,0)^{T}$ \\
\hline Inputs saturations & $u_{i, m a x}$ & $45\left[\mathrm{mT} \cdot \mathrm{m}^{-1}\right]$ \\
\hline Controller gains & $k_{1}$ & 15 \\
\cline { 2 - 3 } & $k_{2}$ & 30 \\
\hline Observer gains & $L$ & 5 \\
\cline { 2 - 3 } & $g_{1}, g_{2}, g_{3}, g_{4}$ & $-6,-13,-12,-4$ \\
\hline
\end{tabular}

reference trajectory.

\section{CONCLUSION}

This paper relies on a fine modeling of a MRI-guided microrobot, and its interaction with the non-Newtonian blood flow, with consideration of wall effects and interactions. This 2D model can be extended mutatis mutandis to $3 \mathrm{D}$, since we deal with projection on vertical axis (thus encompassing the demanding levitation problem) as well as on one of the horizontal axis. An optimal reference trajectory can hence be deduced from the forces balance, so as to minimize the control efforts. We then synthesize a Lyapunov stabilizing control law for the non linear model using a backstepping approach, combined with a high gain observer so as to both rebuild the unmeasured velocities and smooth the noised measurements. On the one hand, simulations illustrate the stability and robustness to noise of the controller observer pair; on the other hand, even if the 

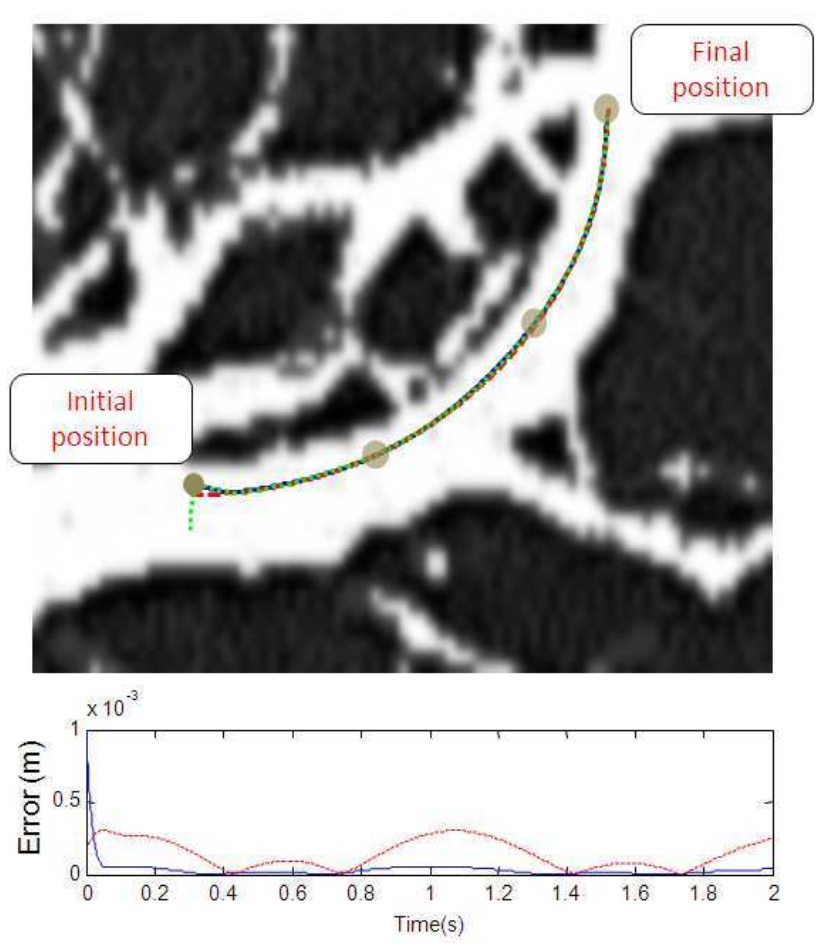

Fig. 5. (a) XZ trajectory: reference trajectory (red dashed line), real trajectory (blue solid line) and estimated trajectory (green dotted line). (b) Error: tracking error (red dashed line) estimation error (blue solid line)

stability is preserved, simulations pinpoint the sensitivity to uncertain physiological parameters.

This sensitivity to matched uncertainties is a challenging issue: if biological parameters are very changeant among patients, the pumping blood is also very difficult to estimate (amplitude, mean value and frequency). If some parameters can be estimated using adaptative backstepping we are working on, like the robot's charge or Hamaker constant, it will not be as simple for parameters like the viscosity. In fact, drag force is a non linear function of viscosity, thus breaking classical adaptative approaches' assumptions, and this problem remains open. We are also working on the difficult problem of estimating the blood velocity, assumed to be known at the moment, using Kazantis-Kravaris Luenberger observers [20]. Finally, the modeling of impact of major bifurcations on the blood's velocity profile is underway. These improvements should robustify the presented approach, and widen the range of application of targeted chemotherapy.

\section{REFERENCES}

[1] U. Kraus-Tiefenbacher et al., "Intraoperative radiotherapy for breast cancer using intrabeam system," Tumori, vol. 91, no. 4, pp. 339-345, 2005.

[2] E. Sievers et al., "Selective ablation of acute myeloid leukemia using antibody-targeted chemotherapy: a phase 1 study of an anti-cd33 calicheamicin immunoconjugate," Blood, vol. 97, no. 11, pp. 36783684, 1999.

[3] G. T. Gillies, R. C. Ritter, W. C. Broaddus, M. S. Grady, M. A. Howard, and R. G. McNeil, "Magnetic manipulation instrumentation for medical physics research," Rev. of Sci. Instruments, vol. 65, no. 3, March 1994.

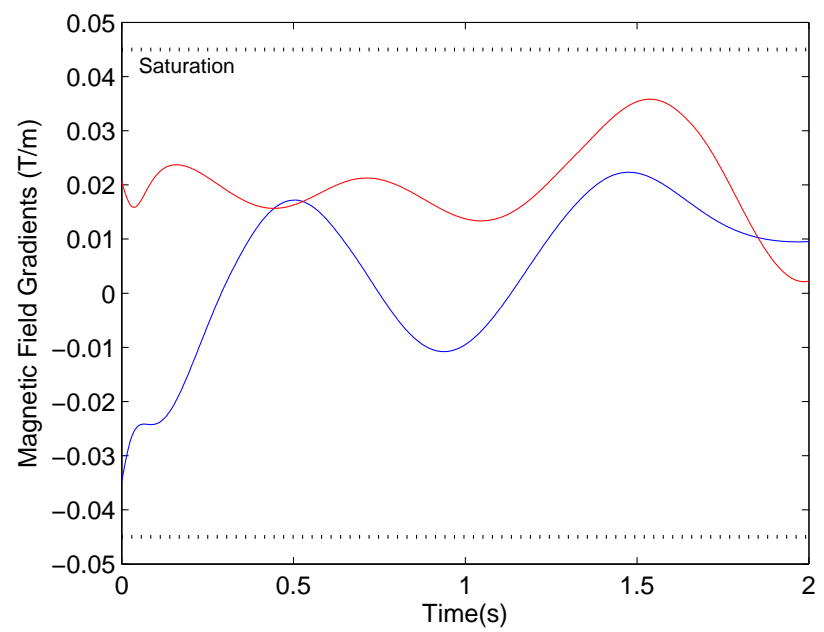

Fig. 6. Control input : magnetic field gradients on $\vec{i}$-axis (red) and on $\vec{k}$-axis (blue)

[4] T. Honda, K. I. Arai, and K. Ishiyama, "Micro swimming mechanisms propelled by external magnetic fields," IEEE Trans. on Magnetics, vol. 32 , no. 5,1996

[5] J. Abbott, Z. Nagy, F. Beyeler, and B. Nelson, "Robotics in the small: Part 1 microrobotics," IEEE Robotics and Automation Magazine, vol. 14, no. 2, pp. 92-103, June 2007.

[6] J.-B. Mathieu, G. Beaudoin, and S. Martel, "Method of propulsion of a ferromagnetic core in the cardiovascular system through magnetic gradients generated by an mri system," IEEE Trans. on Biomedical Engineering, vol. 53, no. 2, pp. 292-299, 2006.

[7] S. Tamaz, R. Gourdeau, A. Chanu, J.-B. Mathieu, and S. Martel, "Real-time mri-based control of a ferromagnetic core for endovascular navigation," IEEE Trans. on Biomed. Eng., vol. 55, no. 7, July 2008.

[8] R. Bawa, "Nanoparticle-based therapeutics in humans : A survey," Nanotechnology Law \& Business, vol. 5:(2), pp. 135-155, 2008.

[9] P. Vartholomeos and C. Mavroidis, "Simulation platform for selfassembly structures in mri-based nanorobotic drug delivery systems," in IEEE. Int. Conf. on Robotics and Automation, May 2010, to be published.

[10] R. Kehlenbeck and R. D. Felice, "Empirical relationships for the terminal settling velocity of spheres un cylindrical columns," Chemical Eng. Technology, vol. 21, pp. 303-308, 1999.

[11] F. White, Viscous Fluid Flow. McGraw Hill New-York, 1991

[12] A.-R. Pries, T.-W. Secomb, and P. Gaehtgens, "Biophysical aspects of blood flow in the microvasculature," Cardiovascular Research, vol. 32(4), pp. 654-667, 1996.

[13] K. Iimura, S. Watanabe, M. Suzuki, M. Hirota, and K. Higashitani, "Simulation of entrainment of agglomerates from plate surfaces by shear flows," Chemical Engineering Science, vol. 64, pp. 1455-1461, 2009.

[14] D. Hays, "Electrostatic adhesion of non-uniformly charged dielectric sphere," Int. Phys. Conf. ser., vol. 118, pp. 223-228, 1991.

[15] - Role of Electrostatics in Adhesion, in Fundamentals of Adhesion, L.-H. Lee, Ed. PLENUM PRESS, 1991.

[16] L. Arcese, A. Cherry, M. Fruchard, and A. Ferreira, "Dynamic behavior investigation for trajectory control of a microrobot in blood vessels," in IROS, 2010, to be published.

[17] M. Krstić, I. Kanellakopoulos, and P. Kokotović, Nonlinear and Adaptative Control Design. John Wiley \& Sins, Inc., New York, 1995.

[18] I. Kanellakopoulos, P. Kokotović, and A. Morse, "A toolkit for nonlinear feedback design," SCL, vol. 18, pp. 83-92, 1992.

[19] J. Gauthier, H. Hammouri, and S. Othman, "A simple observer for nonlinear systems. application to bioreactors." ITAC, vol. 37, no. 6 , pp. 875-880, 1992.

[20] V. Andrieu and L. Praly, "On the existence of a kazantis-kravaris / luenberger observer,” SIAM, vol. 45, no. 2, pp. 432-456, 2006. 